\title{
Tarski Geometry Axioms - Part II
}

\author{
Roland Coghetto \\ Rue de la Brasserie 5 \\ 7100 La Louvière \\ Belgium
}

\author{
Adam Grabowski \\ Institute of Informatics \\ University of Białystok \\ Ciołkowskiego 1M, 15-245 Białystok \\ Poland
}

Summary. In our earlier article [12, the first part of axioms of geometry proposed by Alfred Tarski [14] was formally introduced by means of Mizar proof assistant [9]. We defined a structure TarskiPlane with the following predicates:

- of betweenness between (a ternary relation),

- of congruence of segments equiv (quarternary relation),

which satisfy the following properties:

- congruence symmetry (A1),

- congruence equivalence relation (A2),

- congruence identity (A3),

- segment construction (A4),

- $\operatorname{SAS}(\mathrm{A} 5)$,

- betweenness identity (A6),

- Pasch (A7).

Also a simple model, which satisfies these axioms, was previously constructed, and described in [6]. In this paper, we deal with four remaining axioms, namely:

- the lower dimension axiom (A8),

- the upper dimension axiom (A9),

- the Euclid axiom (A10),

- the continuity axiom (A11).

They were introduced in the form of Mizar attributes. Additionally, the relation of congruence of triangles cong is introduced via congruence of sides (SSS).

In order to show that the structure which satisfies all eleven Tarski's axioms really exists, we provided a proof of the registration of a cluster that the Euclidean 
plane, or rather a natural [5] extension of ordinary metric structure Euclid 2 satisfies all these attributes.

Although the tradition of the mechanization of Tarski's geometry in Mizar is not as long as in Coq 11, first approaches to this topic were done in Mizar in 1990 [16] (even if this article started formal Hilbert axiomatization of geometry, and parallel development was rather unlikely at that time [8]). Connection with another proof assistant should be mentioned - we had some doubts about the proof of the Euclid's axiom and inspection of the proof taken from Archive of Formal Proofs of Isabelle [10] clarified things a bit. Our development allows for the future faithful mechanization of [13] and opens the possibility of automatically generated Prover9 proofs which was useful in the case of lattice theory [7.

MSC: 51A05 51M04 03B35

Keywords: Tarski's geometry axioms; foundations of geometry; Euclidean plane MML identifier: GTARSKI2, version: 8.1.05 5.38.1280

\section{Preliminaries}

Now we state the propositions:

(1) Let us consider real numbers $r, s, t, u$. Suppose $s \neq 0$ and $t \neq 0$ and $r^{2}=s^{2}+t^{2}-2 \cdot s \cdot t \cdot u$. Then $u=\frac{r^{2}-s^{2}-t^{2}}{-2 \cdot s \cdot t}$.

(2) Let us consider a natural number $n$, and elements $u, v$ of $\mathcal{E}_{\mathrm{T}}^{n}$. Then $u+0 \cdot v=u$.

(3) Let us consider a natural number $n$, real numbers $r$, $s$, and elements $u$, $v, w$ of $\mathcal{E}_{\mathrm{T}}^{n}$. If $r \cdot u-r \cdot v=s \cdot w-s \cdot u$, then $(r+s) \cdot u=r \cdot v+s \cdot w$. The theorem is a consequence of (2).

(4) Let us consider real numbers $r$, $s$. If $0<r$ and $0<s$, then $0 \leqslant \frac{r}{r+s} \leqslant 1$.

(5) Let us consider a real number $a$. Then $\cos (3 \cdot \pi-a)=-\cos a$.

Let us consider a natural number $n$ and elements $a, b, c$ of $\mathcal{E}_{\mathrm{T}}^{n}$. Now we state the propositions:

(6) If $a-c=b-c$, then $a=b$.

(7) $c-a-(b-a)=c-b$.

(8) Let us consider real numbers $a, b, c, d$. Then $\rho([a, b],[c, d])=$ $\sqrt{(a-c)^{2}+(b-d)^{2}}$.

(9) $\rho([0,0],[1,0])=1$. The theorem is a consequence of $(8)$.

(10) $\rho([0,0],[0,1])=1$. The theorem is a consequence of $(8)$.

(11) $\rho([1,0],[0,1])=\sqrt{2}$. The theorem is a consequence of $(8)$.

Let $n$ be a natural number. The functor TarskiEuclidSpace $n$ yielding a metric Tarski structure is defined by the term 
(Def. 1) the naturally generated Tarski extension of $\mathcal{E}^{n}$.

The functor TarskiEuclid2Space yielding a metric Tarski structure is defined by the term

(Def. 2) TarskiEuclidSpace 2.

\section{Basic Properties of the Euclidean Plane}

Let $n$ be a natural number. Let us observe that TarskiEuclidSpace $n$ is non empty and TarskiEuclid2Space is reflexive, symmetric, and discernible.

Let $n$ be a natural number. One can check that TarskiEuclidSpace $n$ is reflexive, symmetric, and discernible.

Let $P$ be a point of TarskiEuclidSpace $n$. The functor $\hat{P}$ yielding an element of $\mathcal{E}_{\mathrm{T}}^{n}$ is defined by the term

(Def. 3) $P$.

Let $P$ be a point of TarskiEuclid2Space. The functor $\hat{P}$ yielding an element of $\mathcal{E}_{\mathrm{T}}^{2}$ is defined by the term

(Def. 4) $P$.

The functor $\tilde{P}$ yielding a point of $\mathcal{E}^{2}$ is defined by the term

(Def. 5) $P$.

The functor $\breve{P}$ yielding an element of $\mathcal{R}^{2}$ is defined by the term

(Def. 6) $P$.

Now we state the propositions:

(12) Let us consider a natural number $n$, points $p, q$ of TarskiEuclidSpace $n$, and elements $p_{1}, q_{1}$ of $\mathcal{E}_{\mathrm{T}}^{n}$. Suppose $p=p_{1}$ and $q=q_{1}$. Then

(i) $\rho(p, q)=\rho^{n}\left(p_{1}, q_{1}\right)$, and

(ii) $\rho(p, q)=\left|p_{1}-q_{1}\right|$.

(13) Let us consider points $a, b, c$ of TarskiEuclid2Space. Then $(\rho(c, a))^{\mathbf{2}}=$ $(\rho(a, b))^{2}+(\rho(b, c))^{2}-2 \cdot \rho(a, b) \cdot \rho(b, c) \cdot \cos \measuredangle(\hat{a}, \hat{b}, \hat{c})$. The theorem is a consequence of (12).

(14) Let us consider points $a, b, c, e, f, g$ of TarskiEuclid2Space. Suppose $\hat{a}, \hat{b}, \hat{c}$ form a triangle and $\measuredangle(\hat{a}, \hat{b}, \hat{c})<\pi$ and $\measuredangle(\hat{e}, \hat{c}, \hat{a})=\frac{\measuredangle(\hat{b}, \hat{c}, \hat{a})}{3}$ and $\measuredangle(\hat{c}, \hat{a}, \hat{e})=\frac{\measuredangle(\hat{c}, \hat{a}, \hat{b})}{3}$ and $\measuredangle(\hat{a}, \hat{b}, \hat{f})=\frac{\measuredangle(\hat{a}, \hat{b}, \hat{c})}{3}$ and $\measuredangle(\hat{f}, \hat{a}, \hat{b})=\frac{\measuredangle(\hat{c}, \hat{a}, \hat{b})}{3}$ and $\measuredangle(\hat{b}, \hat{c}, \hat{g})=\frac{\measuredangle(\hat{b}, \hat{c}, \hat{a})}{3}$ and $\measuredangle(\hat{g}, \hat{b}, \hat{c})=\frac{\measuredangle(\hat{a}, \hat{b}, \hat{c})}{3}$. Then

(i) $\rho(f, e)=\rho(g, f)$, and

(ii) $\rho(f, e)=\rho(e, g)$, and 
(iii) $\rho(g, f)=\rho(e, g)$.

The theorem is a consequence of (12).

(15) Let us consider a natural number $n$, elements $p, q$ of TarskiEuclidSpace $n$, and elements $p_{1}, q_{1}$ of $\mathcal{E}^{n}$. If $p=p_{1}$ and $q=q_{1}$, then $\rho(p, q)=\rho\left(p_{1}, q_{1}\right)$.

(16) Let us consider points $p, q$ of TarskiEuclid2Space.

Then $\rho(p, q)=\sqrt{\left((\hat{p})_{\mathbf{1}}-(\hat{q})_{\mathbf{1}}\right)^{\mathbf{2}}+\left((\hat{p})_{\mathbf{2}}-(\hat{q})_{\mathbf{2}}\right)^{\mathbf{2}}}$.

(17) Let us consider points $A, B$ of TarskiEuclid2Space. Then

(i) $\rho(A, B)=|\hat{A}-\hat{B}|$, and

(ii) $\rho(A, B)=|\breve{A}-\breve{B}|$.

(18) Let us consider points $a, b, c, d$ of TarskiEuclid2Space. Then $|\hat{a}-\hat{b}|=$ $|\hat{c}-\hat{d}|$ if and only if $\overline{a b} \cong \overline{c d}$. The theorem is a consequence of (17).

(19) Let us consider points $p, q, r$ of TarskiEuclid2Space. Then $p$ is between $q$ and $r$ if and only if $\hat{p} \in \mathcal{L}(\hat{q}, \hat{r})$. The theorem is a consequence of (15).

From now on $n$ denotes a natural number.

Now we state the propositions:

(20) Let us consider points $p, q, r$ of TarskiEuclid2Space. Then $q$ lies between $p$ and $r$ if and only if $\hat{q} \in \mathcal{L}(\hat{p}, \hat{r})$. The theorem is a consequence of (19).

(21) Let us consider points $a, b$ of TarskiEuclid2Space. Then

(i) $a$ lies between $a$ and $b$, and

(ii) $b$ lies between $a$ and $b$.

The theorem is a consequence of (20).

(22) Let us consider points $a, b, c$ of TarskiEuclid2Space. If $b$ lies between $a$ and $c$, then $b$ lies between $c$ and $a$. The theorem is a consequence of (20).

(23) Let us consider points $a, b$ of TarskiEuclid2Space. If $b$ lies between $a$ and $a$, then $a=b$. The theorem is a consequence of (20).

(24) Let us consider points $a, b$ of TarskiEuclid2Space. Then $a=b$ if and only if $\rho(a, b)=0$. The theorem is a consequence of (12).

(25) Let us consider points $a, b, c, d$ of TarskiEuclid2Space. If $\rho(a, b)+$ $\rho(c, d)=0$, then $a=b$ and $c=d$. The theorem is a consequence of (24).

(26) Let us consider points $a, b, c, a_{1}, b_{1}, c_{1}$ of TarskiEuclid2Space. Then $\triangle a b c \cong \triangle a_{1} b_{1} c_{1}$ if and only if $\rho(a, b)=\rho\left(a_{1}, b_{1}\right)$ and $\rho(a, c)=\rho\left(a_{1}, c_{1}\right)$ and $\rho(b, c)=\rho\left(b_{1}, c_{1}\right)$.

(27) Let us consider points $a, b, c$ of TarskiEuclid2Space. Then $b$ lies between $a$ and $c$ if and only if $\rho(a, c)=\rho(a, b)+\rho(b, c)$. 
(28) Let us consider points $a, b, c, d$ of TarskiEuclid2Space. Then $(\rho(a, b))^{2}=$ $(\rho(c, d))^{2}$ if and only if $\overline{a b} \cong \overline{c d}$.

(29) Let us consider a point $a$ of TarskiEuclid2Space. Then $a$ lies between $a$ and $a$.

\section{Ordered Affine Space Generated by $\mathcal{E}_{\mathrm{T}}^{2}$}

Now we state the proposition:

(30) OASpace $\mathcal{E}_{\mathrm{T}}^{2}$ is an ordered affine space.

PROOF: There exist vectors $u, v$ of $\mathcal{E}_{\mathrm{T}}^{2}$ such that for every real numbers $a$, $b$ such that $a \cdot u+b \cdot v=0_{\mathcal{E}_{\mathrm{T}}^{2}}$ holds $a=0$ and $b=0$ by [4, (58), (56), (52)].

Let us consider elements $a, b, c$ of OASpace $\mathcal{E}_{\mathrm{T}}^{2}$. Now we state the propositions:

(31) $b$ is a midpoint of $a, c$ if and only if $a=b$ or $b=c$ or there exist points $u, v$ of $\mathcal{E}_{\mathrm{T}}^{2}$ such that $u=a$ and $v=c$ and $b \in \mathcal{L}(u, v)$. The theorem is a consequence of (3), (4), (30), and (2).

(32) $b$ is a midpoint of $a, c$ if and only if there exist points $u, v$ of $\mathcal{E}_{\mathrm{T}}^{2}$ such that $u=a$ and $v=c$ and $b \in \mathcal{L}(u, v)$. The theorem is a consequence of (31).

(33) Let us consider elements $a, b, c$ of OASpace $\mathcal{E}_{\mathrm{T}}^{2}$, and points $a_{1}, b_{1}, c_{1}$ of TarskiEuclid2Space. Suppose $a=a_{1}$ and $b=b_{1}$ and $c=c_{1}$. Then $b$ is a midpoint of $a, c$ if and only if $b_{1}$ lies between $a_{1}$ and $c_{1}$. The theorem is a consequence of (32) and (20).

\section{Eucldiean Plane Satisfies First 7 Tarski's Axioms}

Let us consider elements $A, B, C, D$ of $\mathcal{E}_{\mathrm{T}}^{2}$. Now we state the propositions:

(34) If $B \in \mathcal{L}(A, C)$ and $C \in \mathcal{L}(A, D)$, then $B \in \mathcal{L}(A, D)$.

(35) If $B \neq C$ and $B \in \mathcal{L}(A, C)$ and $C \in \mathcal{L}(B, D)$, then $C \in \mathcal{L}(A, D)$. The theorem is a consequence of $(30)$ and (32).

(36) Let us consider points $p, q, r, s$ of TarskiEuclid2Space. If $q$ lies between $p$ and $r$ and $r$ lies between $p$ and $s$, then $q$ lies between $p$ and $s$. The theorem is a consequence of (20) and (34).

(37) Let us consider points $A, B, C, D$ of $\mathcal{E}_{\mathrm{T}}^{2}$. If $B \in \mathcal{L}(A, C)$ and $D \in$ $\mathcal{L}(A, B)$, then $B \in \mathcal{L}(D, C)$. The theorem is a consequence of (34).

Let us consider points $p, q, r, s$ of TarskiEuclid2Space. Now we state the proposition: 
(38) If $q$ lies between $p$ and $r$ and $s$ lies between $p$ and $q$, then $q$ lies between $s$ and $r$. The theorem is a consequence of (20) and (37).

Let us assume that $q \neq r$ and $q$ lies between $p$ and $r$ and $r$ lies between $q$ and $s$. Now we state the propositions:

(39) $q$ lies between $p$ and $s$. The theorem is a consequence of (20) and (35).

(40) $r$ lies between $p$ and $s$. The theorem is a consequence of (20) and (35).

Note that TarskiEuclid2Space satisfies the axiom of congruence symmetry, the axiom of congruence equivalence relation, the axiom of congruence identity, the axiom of segment construction, the axiom of SAS, the axiom of betweenness identity, and the axiom of Pasch and TarskiEuclid2Space satisfies seven Tarski's geometry axioms.

\section{Preparation for The Rest of Tarski's Axioms}

Now we state the propositions:

(41) Let us consider points $P, Q, R$ of $\mathcal{E}_{\mathrm{T}}^{2}$, and an element $L$ of $\operatorname{Lines}\left(\mathcal{R}^{2}\right)$. If $P, Q, R \in L$, then $P \in \mathcal{L}(Q, R)$ or $Q \in \mathcal{L}(R, P)$ or $R \in \mathcal{L}(P, Q)$.

(42) Let us consider elements $a, b, c$ of TarskiEuclid2Space. Suppose $\hat{b} \in$ $\mathcal{L}(\hat{a}, \hat{c})$. Then there exists a real number $r$ such that

(i) $0 \leqslant r \leqslant 1$, and

(ii) $\hat{b}-\hat{a}=r \cdot(\hat{c}-\hat{a})$.

(43) Let us consider a natural number $n$, and elements $a, b, c$ of TarskiEuclidSpace $n$. Suppose $\hat{b} \in \mathcal{L}(\hat{a}, \hat{c})$. Then there exists a real number $r$ such that

(i) $0 \leqslant r \leqslant 1$, and

(ii) $\hat{b}-\hat{a}=r \cdot(\hat{c}-\hat{a})$.

(44) Let us consider elements $a, b, c$ of TarskiEuclid2Space. Suppose there exists a real number $r$ such that $0 \leqslant r \leqslant 1$ and $\hat{b}-\hat{a}=r \cdot(\hat{c}-\hat{a})$. Then $\hat{b} \in \mathcal{L}(\hat{a}, \hat{c})$.

\section{Four Remaining Axioms of TArski}

Let $S$ be a Tarski plane. We say that $S$ satisfies (A8) if and only if

(Def. 7) there exist points $a, b, c$ of $S$ such that $b$ does not lie between $a$ and $c$ and $c$ does not lie between $b$ and $a$ and $a$ does not lie between $c$ and $b$.

We say that $S$ satisfies (A9) if and only if 
(Def. 8) for every points $a, b, c, p, q$ of $S$ such that $p \neq q$ and $\overline{a p} \cong \overline{a q}$ and $\overline{b p} \cong \overline{b q}$ and $\overline{c p} \cong \overline{c q}$ holds $b$ lies between $a$ and $c$ or $c$ lies between $b$ and $a$ or $a$ lies between $c$ and $b$.

We say that $S$ satisfies (A10) if and only if

(Def. 9) for every points $a, b, c, d, t$ of $S$ such that $d$ lies between $a$ and $t$ and $d$ lies between $b$ and $c$ and $a \neq d$ there exist points $x, y$ of $S$ such that $b$ lies between $a$ and $x$ and $c$ lies between $a$ and $y$ and $t$ lies between $x$ and $y$.

We say that $S$ satisfies (A11) if and only if

(Def. 10) for every subsets $X, Y$ of $S$ such that there exists a point $a$ of $S$ such that for every points $x, y$ of $S$ such that $x \in X$ and $y \in Y$ holds $x$ lies between $a$ and $y$ there exists a point $b$ of $S$ such that for every points $x$, $y$ of $S$ such that $x \in X$ and $y \in Y$ holds $b$ lies between $x$ and $y$.

We introduce the notation $S$ satisfies Lower Dimension Axiom as a synonym of $S$ satisfies (A8) and $S$ satisfies Upper Dimension Axiom as a synonym of $S$ satisfies (A9) and $S$ satisfies Euclid Axiom as a synonym of $S$ satisfies (A10) and $S$ satisfies Continuity Axiom as a synonym of $S$ satisfies (A11).

Now we state the proposition:

(45) LOWER DIMENSION AXIOM:

There exist points $a, b, c$ of TarskiEuclid2Space such that

(i) $b$ does not lie between $a$ and $c$, and

(ii) $c$ does not lie between $b$ and $a$, and

(iii) $a$ does not lie between $c$ and $b$.

Proof: Reconsider $a=[0,0], b=[1,0], c=[0,1]$ as a point of TarskiEuclid2Space. $b$ does not lie between $a$ and $c$ by (20), [3, (12)], [15, (19)], (9). $c$ does not lie between $b$ and $a$ by (20), [3, (12)], [15, (19)], (9). $\hat{a} \in \mathcal{L}(\hat{c}, \hat{b})$.

(46) UPPER DIMENSION AXIOM:

Let us consider points $a, b, c, p, q$ of TarskiEuclid2Space. Suppose $p \neq q$ and $\overline{a p} \cong \overline{a q}$ and $\overline{b p} \cong \overline{b q}$ and $\overline{c p} \cong \overline{c q}$. Then

(i) $b$ lies between $a$ and $c$, or

(ii) $c$ lies between $b$ and $a$, or

(iii) $a$ lies between $c$ and $b$.

The theorem is a consequence of (18), (41), and (20).

(47) Axiom of EuClid:

Let us consider elements $a, b, c, d, t$ of TarskiEuclid2Space. Suppose $d$ lies between $a$ and $t$ and $d$ lies between $b$ and $c$ and $a \neq d$. Then there exist elements $x, y$ of TarskiEuclid2Space such that 
(i) $b$ lies between $a$ and $x$, and

(ii) $c$ lies between $a$ and $y$, and

(iii) $t$ lies between $x$ and $y$.

Proof: $\hat{d} \in \mathcal{L}(\hat{a}, \hat{t})$. Set $v=\hat{a}$. Set $w=\hat{t}$. Consider $r$ being a real number such that $0 \leqslant r \leqslant 1$ and $\hat{d}=(1-r) \cdot v+r \cdot w$. Set $r_{1}=\frac{1}{r} \cdot r \neq 0$ by [17, (10), (21)]. Set $x_{1}=r_{1} \cdot(\hat{b}-\hat{a})+\hat{a}$. Reconsider $x_{2}=x_{1}$ as an element of TarskiEuclid2Space. $\hat{b} \in \mathcal{L}\left(\hat{a}, \hat{x_{2}}\right)$. $b$ lies between $a$ and $x_{2}$. Set $y_{1}=r_{1} \cdot(\hat{c}-\hat{a})+\hat{a}$. Reconsider $y_{2}=y_{1}$ as an element of TarskiEuclid2Space. $\hat{c} \in \mathcal{L}\left(\hat{a}, \hat{y}_{2}\right) . c$ lies between $a$ and $y_{2} . \hat{d} \in \mathcal{L}(\hat{b}, \hat{c})$. Consider $k$ being a real number such that $0 \leqslant k \leqslant 1$ and $\hat{d}-\hat{b}=k \cdot(\hat{c}-\hat{b}) . \hat{t} \in \mathcal{L}\left(\hat{x_{2}}, \hat{y_{2}}\right)$. $t$ lies between $x_{2}$ and $y_{2}$.

\section{Axiom A11 - Axiom Schema of Continuity}

Now we state the proposition:

(48) Axiom schema of Continuity:

Let us consider subsets $X, Y$ of TarskiEuclid2Space. Suppose there exists an element $a$ of TarskiEuclid2Space such that for every elements $x, y$ of TarskiEuclid2Space such that $x \in X$ and $y \in Y$ holds $x$ lies between $a$ and $y$. Then there exists an element $b$ of TarskiEuclid2Space such that for every elements $x, y$ of TarskiEuclid2Space such that $x \in X$ and $y \in Y$ holds $b$ lies between $x$ and $y$. The theorem is a consequence of (20), (42), (2), and (44).

Let us observe that TarskiEuclid2Space satisfies Lower Dimension Axiom, Upper Dimension Axiom, Euclid Axiom, and Continuity Axiom.

\section{Corrolaries}

In the sequel $X, Y$ denote subsets of TarskiEuclid2Space.

Let us consider an element $a$ of TarskiEuclid2Space. Now we state the propositions:

(49) Suppose for every elements $x, y$ of TarskiEuclid2Space such that $x \in X$ and $y \in Y$ holds $x$ lies between $a$ and $y$ and $a \in Y$. Then

(i) $X=\{a\}$, or

(ii) $X$ is empty. 
(50) Suppose for every elements $x, y$ of TarskiEuclid2Space such that $x \in X$ and $y \in Y$ holds $x$ lies between $a$ and $y$ and $X$ is not empty and $Y$ is not empty and if $X$ is trivial, then $X \neq\{a\}$. Then there exists an element $b$ of TarskiEuclid2Space such that

(i) $X \subseteq \operatorname{Line}(\hat{a}, \hat{b})$, and

(ii) $Y \subseteq \operatorname{Line}(\hat{a}, \hat{b})$.

Proof: Consider $x_{0}$ being an object such that $x_{0} \in X$. Consider $c$ being an object such that $c \in Y . X \subseteq \mathcal{L}(\hat{a}, \hat{c}) . Y \subseteq \operatorname{Line}(\hat{a}, \hat{c})$ by [2, (131)], (20), [1, (73), (72), (75)].

\section{REFERENCES}

[1] Czesław Byliński. Introduction to real linear topological spaces. Formalized Mathematics, 13(1):99-107, 2005.

[2] Czesław Byliński. Some basic properties of sets Formalized Mathematics, 1(1):47-53, 1990.

[3] Roland Coghetto. Circumcenter, circumcircle and centroid of a triangle. Formalized Mathematics, 24(1):17-26, 2016. doi 10.1515/forma-2016-0002.

[4] Agata Darmochwał. The Euclidean space Formalized Mathematics, 2(4):599-603, 1991.

[5] Adam Grabowski. Efficient rough set theory merging. Fundamenta Informaticae, 135(4): 371-385, 2014. doi $10.3233 /$ FI-2014-1129.

[6] Adam Grabowski. Tarski's geometry modelled in Mizar computerized proof assistant. In Proceedings of the 2016 Federated Conference on Computer Science and Information Systems, FedCSIS 2016, Gdańsk, Poland, September 11-14, 2016, pages 373-381, 2016. doi: $10.15439 / 2016 \mathrm{~F} 290$

[7] Adam Grabowski. Mechanizing complemented lattices within Mizar system. Journal of Automated Reasoning, 55:211-221, 2015. doi 10.1007/s10817-015-9333-5.

[8] Adam Grabowski and Christoph Schwarzweller. On duplication in mathematical repositories. In Serge Autexier, Jacques Calmet, David Delahaye, Patrick D. F. Ion, Laurence Rideau, Renaud Rioboo, and Alan P. Sexton, editors, Intelligent Computer Mathematics, 10th International Conference, AISC 2010, 17th Symposium, Calculemus 2010, and 9th International Conference, MKM 2010, Paris, France, July 5-10, 2010. Proceedings, volume 6167 of Lecture Notes in Computer Science, pages 300-314. Springer, 2010. doi $10.1007 / 978-3-642-14128-7 \_26$.

[9] Adam Grabowski, Artur Korniłowicz, and Adam Naumowicz. Four decades of Mizar. Journal of Automated Reasoning, 55(3):191-198, 2015. doi 10.1007/s10817-015-9345-1

[10] Timothy James McKenzie Makarios. A mechanical verification of the independence of Tarski's Euclidean Axiom. 2012. Master's thesis.

[11] Julien Narboux. Mechanical theorem proving in Tarski's geometry. In F. Botana and T. Recio, editors, Automated Deduction in Geometry, volume 4869 of Lecture Notes in Computer Science, pages 139-156. Springer, 2007.

[12] William Richter, Adam Grabowski, and Jesse Alama. Tarski geometry axioms. Formalized Mathematics, 22(2):167-176, 2014. doi 10.2478/forma-2014-0017.

[13] Wolfram Schwabhäuser, Wanda Szmielew, and Alfred Tarski. Metamathematische Methoden in der Geometrie. Springer-Verlag, Berlin, Heidelberg, New York, Tokyo, 1983.

[14] Alfred Tarski and Steven Givant. Tarski's system of geometry. Bulletin of Symbolic Logic, $5(2): 175-214,1999$.

[15] Andrzej Trybulec and Czesław Byliński. Some properties of real numbers Formalized Mathematics, 1(3):445-449, 1990.

[16] Wojciech A. Trybulec. Axioms of incidence. Formalized Mathematics, 1(1):205-213, 1990. 
[17] Wojciech A. Trybulec. Vectors in real linear space. Formalized Mathematics, 1(2):291-296, 1990.

Received June 30, 2016 